\title{
Surgical training system with a Web Portal for virtual environment searching
}

\author{
G. Aloisio, L. T. De Paolis, A. De Mauro \& A. Zuccalà \\ Department of Innovation Engineering \& CACT/ISUFI, \\ Lecce University, Italy
}

\begin{abstract}
Virtual reality based surgical simulators can provide a new method for apprenticeship which is at least as efficient as the traditional one and which could reduce the length of training. It is very important to carry out the same surgical procedure on a variety of different case studies that correspond to several virtual patients, each of them exhibiting a specific difficulty depending on the different anatomy or pathology. In order to obtain a realistic simulation it is necessary to use a specific haptic device which is able to replicate the force feedback sensations relating to the interaction in the virtual environment on the user. The aim of this paper is to present a virtual surgical simulator to perform training on different cases of a specific surgical procedure; the building of the virtual environment is based on images of real patients and a Web Portal is used to search a specific pathologies or anatomic structures; this information are included in the metadata associated with the virtual environment.

Keywords: virtual reality, surgical training simulator, web services, web portal, metadata, haptic interface.
\end{abstract}

\section{Introduction}

Simulators have been used in aviation, nuclear power, military flight operations and other industries as a training tool and method to assess performance; recently the use of simulation in medicine has increased markedly due to greater awareness of the importance of patient safety.

In medical training, simulators can provide a substitute for actual patients and they improve patient safety by allowing physicians to become better trained without putting patients at risk. This training method presents several 
advantages; it provides a simulation of uncommon but critical scenarios in which a rapid response is needed and makes it possible to see the results of some surgical decisions without risks for the patient. Studies of the effectiveness of simulators are in progress in order to measure their performance and to establish a clear link between simulator training and patient outcomes.

Simulators in the surgical setting are aimed at improving surgeons' technical skills and dexterity. Surgical simulators have been developed for a variety of procedures; intuitively, improved technical skills should lead to fewer complications during surgical procedures by improving manual dexterity and preventing adverse events. Problem-based surgical simulation may improve patient safety not only by improving skills, but also by training surgeons how to better anticipate and avoid complications and how to manage them should they occur. Minimally invasive surgical methods require different training from traditional techniques; frequent training should be carried out in a safe environment which mimics the anatomy and physiology of the body as closely as possible to ensure adequate transfer of skills. In addition, these procedures need to be learned by repetition.

Virtual reality is a technology that can teach surgeons about new minimally invasive surgical methods that require the acquisition of new skills and can allow the trainee to return to the same procedure or task several times later as a refresher course. Virtual reality simulation could duplicate the operative field and thereby enhance training and reduce the need for expensive animal training models. This technology makes it possible to model the real behaviour of tissues and medical instruments and to develop a real-time learning environment for surgeons.

\section{Training simulator requirements}

Virtual reality based surgical simulators can provide a new method for apprenticeship which is at least as efficient as the traditional one and which could reduce the length of training. On the other hand the simulator fidelity (how accurately the simulator replicates the reality) can be imperfect and refinement of simulators to make them more sophisticated may help to improve the quality of the training but the proficiency on a simulator does not ensure proficiency in clinical settings.

In simulation-based training there is the potential risk that the simulator does not properly replicate the tasks or task environment of caring for patients; in this case the clinicians might acquire inappropriate behaviours (negative training) or develop a false sense of security in their skills that could theoretically lead to harm. For this reason it is very important to build the virtual environment starting from the real patient's images in order to have a realistic scenario.

A simulation of how the human soft tissue behaves under the effect of external stimulations and real-time interactions with the medical instruments are the essential features of a training simulator and strictly depend on the accuracy of the physical modelling of the tissue and the use of a force feedback device [1]. 
It is very important to carry out the same surgical procedure on a variety of different case studies, studies which differ in terms of the pathology, the anatomical structure and the patient's age, so that they correspond to several virtual patients, each of them exhibiting a particular difficulty depending on the different anatomy or pathology.

In order to guarantee the accuracy and efficiency of the simulation it is absolutely necessary to implement firstly correct collision detection and response algorithms which take into account the fundamental requirement of the real time interactions and secondly a physical modelling algorithm which aims to obtain a computational representation of the deformable organs behaviour.

The use of a specific surgical procedure oriented haptic device able to replicate the force feedback sensations on the user and to provide a manipulation of the medical instruments identical to the real one, improves the immersion sensation in the virtual environment.

The aim of this paper is to present a surgical virtual simulator to perform training on different cases of a specific surgical procedure; the building of the virtual environment is based on real patient's images. Using a Web Portal it is possible to search a virtual environment with specific features in terms of pathology or anatomy; these data are saved on a database with the relevant metadata.

\section{Related works}

\subsection{From medical imaging to virtual reality environments}

In recent years, the biomedical imaging modalities such as Magnetic Resonance Imaging (MRI), Computer Tomography (CT) and Ultrasounds have become essential in identifying, diagnosing and localizing structures and abnormalities. Traditional methods for evaluating data required the viewer to mentally reconstruct a volume from a series of radiographic films or two-dimensional images. The advent of real-time three-dimensional visualization has enabled interactive examination of the internal and external structures from any viewpoint.

RadioDexter 1.0 generates a stereoscopic virtual reality environment in which a user can work interactively with real-time 3D data. RadioDexter processes data from CT or MRI, as well as volumetric ultrasounds. A variety of virtual tools for visualization and surgical planning are accessible while working inside RadioDexter's 3D virtual workspace. This allows the user to work with complex multi-modal imaging data in a fast and intuitive way, for a clear threedimensional understanding of the patient's anatomy and for improved treatment planning [2].

The Virtual Reality in Medicine Lab (VRMedLab) at the University of Chicago uses VR displays developed by the Electronic Visualization Laboratory for their projects. They began with a virtual temporal bone, virtual pelvic floor and virtual craniofacial anatomy to teach their surgical residents as well as share their surgical expertise with other institutions throughout the world. Their 
networked system provides a way of taking the patient's CT data and within 10 minutes have it up on their VR system, available to be reviewed from any place in the world where they have at least a desktop VR system [3].

Freidlin et al. of Center For Information Technology of National Institutes of Health (Bethesda), have been developing NIHmagic, a visualization tool for research and clinical use, on the SGI Onyx II Infinite Reality platform. Images are reconstructed into a three-dimensional volume-by-volume rendering, a display technique that employs three-dimensional texture mapping to give the object a translucent appearance. A stack of slices is rendered into a volume by an opacity mapping function, where the opacity is determined by the intensity of the voxel and its distance from the viewer. NIHmagic incorporates the 3D visualization of time-sequenced images, the manual registration of $2 \mathrm{D}$ slices, the segmentation of anatomical structures and the coloured coded remapping of intensities [4].

\subsection{Web services for image searching}

Several projects deal with developing data management architectures using the grid and web services technologies.

The Biomedical Informatics Research Network (BIRN) creates a long term and robust infrastructure for data sharing and collaboration in biomedical science on a large scale (15 Universities and 22 Research Groups connected through Internet2). A processing pipeline has been developed to analyze and mine MR images acquired at multiple sites using processing and visualization tools developed at multiple sites [5].

At ICM (Interdisciplinary Centre for Mathematical and Computational Modelling), Warsaw University, Poland, a general framework for implementing a wide range of user applications has been developed; in order to support users with dedicated biomolecular services, a BioGRID portal that provides information on resources available, together with description of models, codes and links to most relevant related materials, has been set up and it is being further developed [6].

The European Grid of Solar Observations (EGSO) is employing the grid computing concepts to federate heterogeneous solar data archives into a single virtual archive, allowing scientists to easily locate and retrieve particular data set within the grid. The web services have been used in EGSO as a means of communicating between the various roles in the system [7].

Liu et al. have developed a unified referral information system in which patient care records can be shared among hospitals over the Internet. The XMLbased medical records enable a computer to capture the meaning and structure of the document on the web [8].

TACWeb is a web-based grid portal developed for the management of the biomedical images in a distributed environment. Building on top of the Globus Toolkit, it is an interactive environment that deals with complex user's requests, such as the processing and delivering of biomedical images using the power and security of computational grids. The web services approach is exploited in the architecture design [9]. 


\section{System description}

Grid computing is an emerging technology which makes it possible to share resources such as computational power, high performance devices, data and database. We are developing a service-oriented architecture that wraps virtual medical environments and data application as Web services. These services are capable of satisfying multiple client requests at the same time. Additionally, since Web services are language and platform agnostic, they are easily accessible by clients written in different languages on disparate platforms.

Standards such as XML and SOAP ensure interoperability, UDDI and WSDL allow the discovery and description of Web services.

This architecture makes it possible to search virtual environments built from real patient's medical images located in geographically remote medical centres and to download this data in order to be integrated in the local training simulator; this happens independently of the medical centre where the data has been generated. Due to the complexity of the data stored in the medical centres databases, the searching of the desired virtual environment is based on the descriptive information stored with the data (metadata).

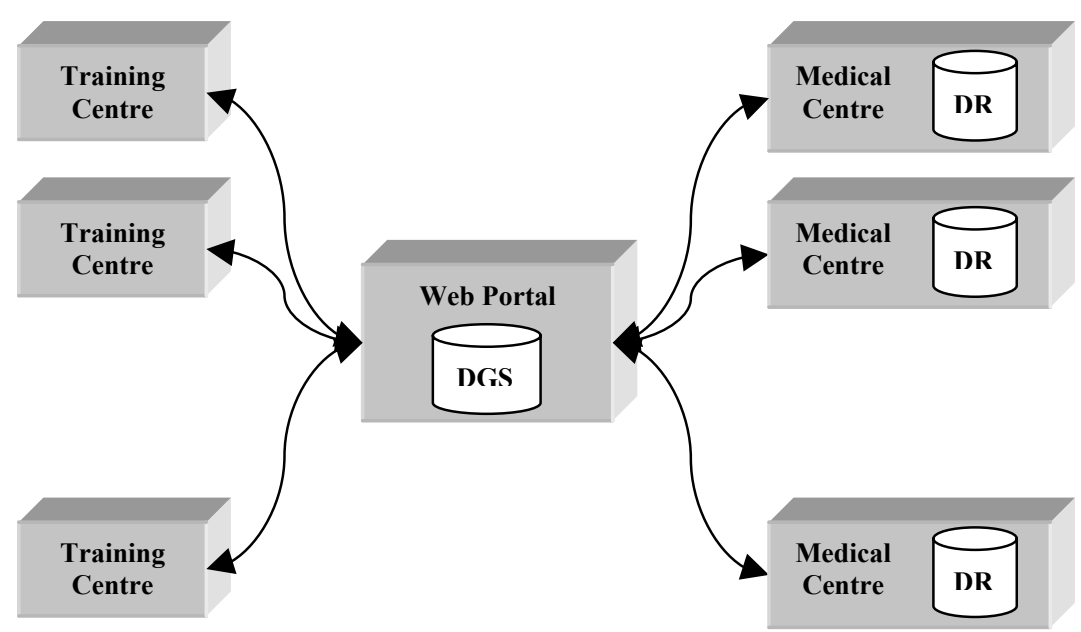

Figure 1: Grid Platform for Virtual Environment Searching.

The scenario is shown in Figure 1 where the main components of the system are:

- Training Centre (TC) where the user can perform training on the different surgical procedures and where the specific haptic devices are available;

- Data Gather Server (DGS) where metadata of the virtual environment of different medical centres are collected; 
- Medical Centre (MC) which provides the access to the local Data Repository (DR);

- Data Repository (DR) where the different virtual environments are physically contained with the relevant metadata.

The functional architecture of the system defines several Medical Centres (MCs) in which virtual environment built from the medical images and related metadata with specific features in terms of pathology or anatomy are stored. An upgraded list of the metadata is present on the web portal and indicates the right Medical Centre where the virtual environment is stored.

The web portal is written using JSP technology and it interacts with the MCs using the Web Services, the fundamental building blocks in Internet distributed computing [10]. In this way the data exchanges between MCs and web portal are made automatically when new data are generated in a $\mathrm{MC}$; medical data are collected in a centralized database localized on the web portal. The implementation details of the service are hidden, so the services can be used on a platform-independent basis. XML is used to define general objects in a intuitive format and to specify resources in portals [11].

The system has two different users:

- a consumer (surgeon, doctor or trainer colleague) that interacts with the centralized and updated database (DGS) using the web portal in order to download a specific virtual environment;

- an administrator that operates in each $\mathrm{MC}$ in order to update the local database with new provided data.

Each Training Centre is provided with a specific haptic device and with the software necessary both to manage the interaction in the virtual environment (collision detection and response algorithms) and to obtain realistic deformations of the organs (physical modelling algorithm).

The searched data are downloaded from the Medical Centre to the Training Centre using a compression technique based on Edgebreaker algorithm, that is a method for compressing 3D data sets and specifically triangle meshes [12].

After the integration of the virtual environment in the simulation system it is possible to perform training on the specific surgical procedures.

The proposed system exploits a 3-tiers architecture [13]:

- a trainee tier where the search starts using a browser and the decompression of the virtual environment happens;

- a middle tier where the web portal is located with the list of metadata;

- back-end services where the building of the virtual environment is carried out and the data repository are located.

\section{Virtual environment building}

Recently, the use of digital images for medical diagnosis has increased considerably. New and better applications are therefore needed in order to effectively manage such information. To build a virtual environment from the real patients' images, the geometric models of the human organs have been reconstructed using data acquired by a CT scanner; data are processed to 
distinguish the anatomical structures and to associate different chromatic scales to the organs. In order to carry out a high quality recognition of the tissues it is necessary to use the correlative information obtained in the three acquisition phases. Due to the distortion produced by the movement of the organs, the three images have to be aligned, using a morphing algorithm, and then recognized, using a clustering algorithm.

Multispectral classification uses registered 3D image volumes from more than one imaging modality or from different sequences within a modality to classify tissues within those volumes. The complementary information contained within the different image volumes may allow for the separation of tissue class types. The segmentation and classification phases are carried out in order to obtain information about the size and the shape of the human organs [16].

A Region Growing Algorithm has been used in the segmentation phase; whereas the classification phase is a user-driven process [17]. In order to obtain the triangulated model of the organs, the Marching Cubes Algorithm has been used [20]. The data processing procedure is performed in an off-line phase before starting the real-time simulation. This process of virtual environment building has been applied to the abdominal region of a human body in a previous project [19]. Figure 2 shows the different steps necessary to carry out the surgical simulation using a virtual environment built from real patient's CT scan images.

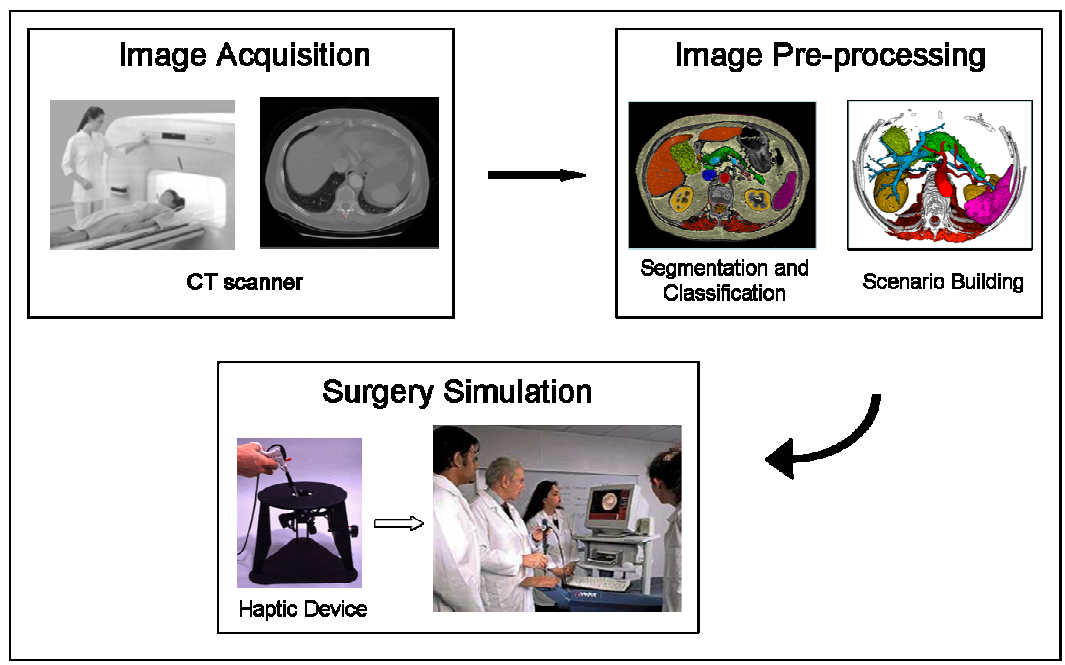

Figure 2: Steps to carry out the surgical simulation.

\section{Training simulator}

A simulation system to perform training on the cathetering procedure has been developed taking into account the results of the HERMES Project (HEmatology 
Research virtual MEdical System) managed by Consorzio CETMA, Brindisi, Italy; the aim of this project is to build an integrated system to simulate an angioplasty intervention [15].

The designed training simulator consists of a virtual environment and a haptic interface, a device able to reproduce on the user the force feedback generated during the interactions in the virtual environment.

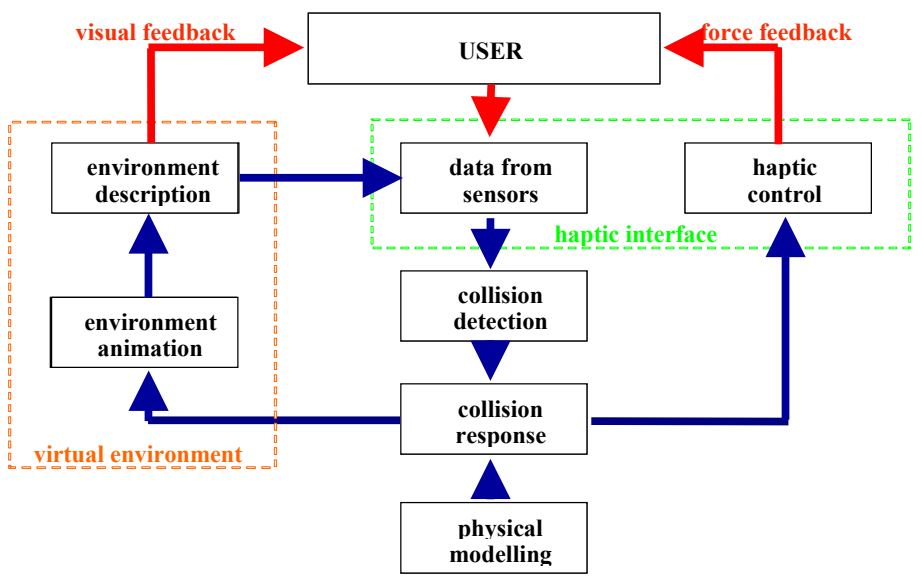

Figure 3: Scheme of the training simulator.

The user interacts with the training system using the haptic interface and data acquired from haptic device sensors are used to represent the instrument position in the virtual environment and to determine possible collisions between virtual objects. Movements of haptic devices lead to changes in the virtual environment representation; collisions between virtual objects produce forces that are replicated on the surgeon's hand by the haptic interface.

The scheme of the simulator is shown in Figure 3.

The haptic interface, designed and built by the PERCRO Laboratory of Scuola Superiore S. Anna, Pisa, Italy, reproduces the real surgical instruments used by the cardiologist. This device is provided with two degrees of freedom which produce force and torque resistance [14]. In particular, as shown in the Figure 4, the system responds to the following forces applied by the user:

- the longitudinal forces in the form of push and pull movements;

- the torque forces in the form of twisting around the longitudinal axis.

Since the arteries are soft tissues, their shape may change during an operation; for this reason physical modelling of the organs is necessary to render their behaviour under the influence of surgeon's instruments. The Finite Element Method (FEM) has been used to model the soft tissues of the artery, using linear elasticity to reduce computational time and speed up interaction rates [21].

In the previously developed simulator the virtual environment has been built using advanced $\mathrm{CAD}$ modelling techniques and taking into account the 
anatomical models described in medical literature. As described in this work, an improvement could be obtained by building the virtual environment from the real patient's images.

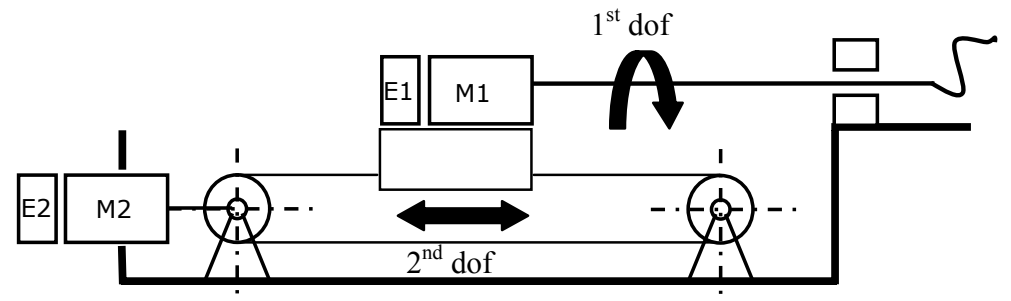

Figure 4: Degrees of freedom of the haptic interface.

\section{Conclusion and future work}

A 3-tier architecture which makes it possible, using a Web Portal, to look for a virtual environment with specific features in terms of pathology or anatomy is presented.

The required data are stored in the databases of several medical centres after performing the different steps to build the virtual environment from real patient's CT scan images. Each virtual environment has the relevant metadata defined in terms of pathology, patient's age, anatomical structures of the organs, etc.; an upgraded list of all metadata is present on the web portal. The Web Services technology is used to interact with the Medical Centres. After downloading the $\mathrm{VE}$ to the training centre, it can be integrated in the surgical simulator to perform training on the cathetering procedure.

The building of the surgical simulator and the implementation of the different steps to obtain the virtual environment have been carried out; an improvement in terms of the visual rendering is obtained building the virtual environment from the real patient's images.

A more accurate definition of the specific metadata is in progress.

Future work will regard the adoption of a correct security policy to exchange data between the training and the medical centres.

\section{References}

[1] Van den Elsen, P. A., Pol, E. J. D., \& Viergever M. A., Medical Image Matching - a Review with Classification, Proc. of the IEEE Engineering in Medicine and Biology, 12(1), 26-39, 1993.

[2] RadioDexter, www.volumeinteractions.com/prod radiodexter.html

[3] VRMedLab, http://www.ahs.uic.edu/ahs/php/index.php? sitename=vrml

[4] Freidlin, R.Z., Ohazama, C.J., Arai, A.E., McGarry, D.P., Panza, J.A.\& Trus , B.L., NIHmagic: 3D Visualization, Registration and Segmentation Tool, 28th AIPR Workshop: 3D Visualization for Data Exploration and Decision Making, Proc of SPIE, Vol. 3905, 2000. 
[5] Beg, M.F., Ceritoglu, C., Kolasny, A.E., Priebe, C.E., Ratnanather, J.T., Yashinski, R., Younes, L., Yu, P., Jovicich, J., Buckner, R.L., Pieper, S., Fischl, B. \& Miller, M.I., Biomedical Informatics Research Network: Multi-site Processing pipeline for shape analysis of brain structures, Proc. of the Human Brain Mapping, Budapest, 2004.

[6] Pytlinski, J., Skorwider, L., Bala, P., Nazaruk, M. \& Wawruch, K., BioGRID - Uniform Platform for Biomolecular Applications, Lecture Notes in Computer Science, Eds. B. Monien, R. Feldman - SpringerVerlag, pp. 881-884, 2002.

[7] Martin, S., Pike, D., Using Web services in the European Grid of Solar Observation (EGSO), Space Science and Technology Department, Rutherford Appleton Laboratory, Chilton, 2003.

[8] Liu, C.T., Long, A. G., Li, Y.C., Tsai, K.C. \& Kuo, H.S., Sharing Patient Care Records over the World Wide Web, International Journal of Medical Informatics, No. 61, pp. 189-205, 2001.

[9] Aloisio, G., Blasi, E., Cafaro, M., Fiore, S., Lezzi, D. \& Mirto, M., Web Services for Biomedical Imaging Portal, Proc. of the ITCC 2003, pp. 432436, 2003.

[10] Kreger, H., Web Services Conceptual Architecture, Armonk et al., 2001.

[11] XML, www.xml.org

[12] Aloisio, G., Barba, M.C., Cafaro, M., Blasi, E., Fiore, S., Mirto, M., Web Services for Edgebreaker Compression in a Grid Portal, Proc. of the HealthGrid Conf., Clermont, France, 2004.

[13] Fox G.C., Portals and Frameworks for Web Based Education and Computational Science, Proc. of the $2^{\text {nd }}$ International Conference on the Practical Application of Java, Omer Rana: Manchester, England, 2000.

[14] Aloisio, G., Bergamasco, M., De Paolis, L.T. et al., Computer-Based Simulator for Catheter Insertion Training, Medicine Meets Virtual Reality 12, Vol. 98, J.D. Westwood et al. (Eds.), IOS Press, pp. 4-6, 2004.

[15] Aloisio, G., De Paolis, L.T., Provenzano, L., An Integrated System for the Angioplasty Intervention Simulation, Proc. of the MEDICON and HEALTH TELEMATICS 2004 Conf., Vol. 6, 2004, Ischia (Naples), Italy, 2004.

[16] Dawson, S., \& Kaufman, J., The Imperative for Medical Simulation, Special Issue on Virtual \& Augmented Reality in Medicine, pp. 479-483, 1998.

[17] Gorman, P. J., Meier, A., \& Krummel, T. M., Simulation and Virtual Reality in Surgical Education, Archives of Surgery, 134, pp.1203-1208, 1998.

[18] Kneoaurek, K., Ivanovic, M., Machac, J., \& Weber, D. A., Medical Image Registration, Europhysics News, 31(4), 2000.

[19] Aloisio, G., Blasi, E., Cafaro, M., Mirto, M. \& Fiore, S., A Genetic Algorithm for Medical Image Registration, Proc. of the SCI2002, Orlando, Florida, Vol XIII, pp.192-197, 2002. 
[20] Lorensen, W., \& Cline, H., Marching Cubes: a High Resolution 3D Surface Construction Algorithm, Proc. of the SIGGRAPH '87, pp. 163$169,1987$.

[21] Aloisio, G., De Paolis, L.T., Mongelli, A., Provenzano, L., Artery SoftTissue Modelling for Stent Implant Training System, Journal of Systemics, Cybernetics and Informatics, 2(4), 2004. 